\title{
Peritoneal inclusion cysts as a diagnostic and treatment challenge
}

\author{
Agata Natkanska' ${ }^{(D}$, Magdalena A. Bizon-Szpernalowska² ${ }^{\circledR}$, \\ Tomasz Milek ${ }^{3}{ }^{-}$, Wlodzimierz Sawicki²] \\ ${ }^{1}$ Scientific Student's Group of Chair and Department of Obstetrics, Gynecology and Gynecological Oncology, Medical University \\ of Warsaw, Poland \\ ${ }^{2}$ Chair and Department of Obstetrics, Gynecology and Gynecological Oncology, Medical University of Warsaw, Poland \\ ${ }^{3} 1^{\text {st }}$ Department and Clinic of General and Vascular Surgery, Medical University of Warsaw, Poland
}

\begin{abstract}
Peritoneal inclusion cysts (PICs) are benign multilocular cysts that consist of fluid localized between intraperitoneal adhesions. They usually present in women in the third and fourth decades of life with a history of prior pelvic or abdominal surgery, especially months to 20 years ago. PICs have low mortality and the potential for high morbidity. Transvaginal ultrasound with color Doppler has an important role in the differential diagnosis. Also, a very important role is played by CA 125 plasma level or use of the ROMA algorithm. But thanks to laparoscopy and the possibility of biopsy from suspicious lesions the correct diagnosis can be established. Treatment of PICs depends individually on the patient's condition, symptoms, other diseases, and desire for procreation. Among other contemplated are hormonal oral contraceptive, an image-guided aspiration, minimally invasive or open surgery. The issue of diagnosis and treatment of PICs requires the continuation of multicentre, randomized clinical trials to find and standardize effective, personalized treatments for PICs.
\end{abstract}

Key words: peritoneal inclusion cysts; PIC; adhesions; infertility; ascites

Ginekologia Polska 2021; 92, 8: 583-586

\section{INTRODUCTION WITH JUSTIFICATION OF THE SELECTED TOPIC}

Peritoneal inclusion cysts (PICs) are benign multilocular cysts that consist of fluid localized between intraperitoneal adhesions. Inflammation, endometriosis, and previous abdominal or pelvic surgery are the risk factors of peritoneal adhesions. Fluid, naturally produced by the peritoneum, becomes trapped inside the peritoneal cavity. When a complex cystic pelvic mass occurs, the patient complains of lower abdominal pain, pelvic fullness, and discomfort in this area [1].

PIC is very difficult to diagnose, resulting in delayed treatment. This article aims to focus on this problem and try to establish the best method of diagnosis and treatment.

\section{DESCRIPTION OF THE CURRENT STATE OF KNOWLEDGE}

Peritoneal inclusion cysts (PICs) are also known as "benign (multi) cystic peritoneal mesothelioma", "inflammatory cysts of the peritoneum", "postoperative peritoneal cyst” or "benign papillary peritoneal cystosis" [2]. They usually present in women in the third and fourth decades of life with a history of prior pelvic or abdominal surgery, especially months to 20 years ago [3]. PICs are complications after pelvic inflammatory disease, endometriosis, and radiotherapy, which allow the creation of adhesions. Also, trauma of the abdomen or inflammatory bowel disease can be a reason for peritoneal inclusion cysts [2, 4]. PICs have low mortality and the potential for high morbidity [3].

Statistics show that PICs occur only after $2 \%$ to $6 \%$ of gynecologic operations, compared to $17 \%$ of male patients with an extensive surgical history [3].

The most common signs and symptoms of PICs are progressive pelvic pain and sometimes palpable abdominal mass. Back pain, dyspareunia, early satiety, constipation, and urinary frequency are less frequently reported. Infertility, which can be a huge problem in patients with PICs, may be correlated with the presence of adhesions [5]. Secondarily 

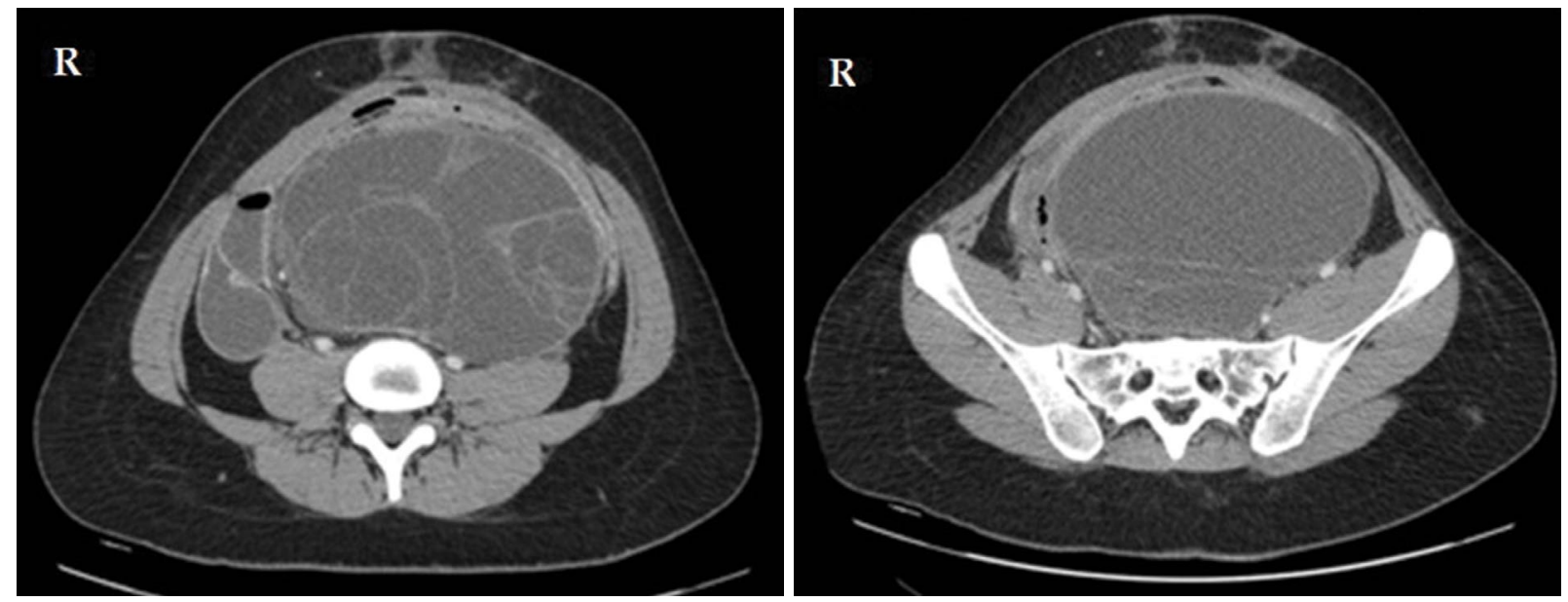

Figure 1. CT scan 11.2014

to compression also pulmonary embolism and venous stasis may occur [2]. On the other hand, up to $10 \%$ of PICs are diagnosed accidentally at the time of imaging or surgery, which highlights the frequency of asymptomatic patients [5].

\section{Diagnosis of PICs}

Diagnosis of PICs is based on visible methods. The ultrasound image of PICs typically is called "spider in the web" pattern. They are described as multilocular cystic lesions with a smooth thin wall. Multiseptate cysts contain liquid, adjacent to pelvic structures and adherent to the surface of the ovary, but not involving the parenchyma of the ovary [6]. These lesions can be similar to a cystic neoplasm, particularly ovarian cancer, for which they may be mistaken. Transvaginal ultrasound with color Doppler has an important role in the differential diagnosis. Also, a very important role is played by CA 125 plasma level or use of the ROMA (risk of ovarian malignancy algorithm), which is calculated using the marker CA 125 and HE4 protein [4]. Magnetic resonance is a helpful tool because of deep visualization and assessment of infiltration of pathological structures [5].

Although laparoscopy and laparotomy often increase the risk of adhesions, which are known as risk factors of PICs, they are also methods of diagnosis and treatment of PICs [4].

The final diagnosis is always based on the histological result. Fluid cytology is usually nonspecific and can lead to misdiagnosis in up to one-third of cases. But thanks to laparoscopy and the possibility of biopsy from suspicious lesions the correct diagnosis can be established [3].

To highlight the importance of the diagnostic and treatment process we would like to present a case of a 30-year-old woman with surgical treatment of familial adenomatous polyposis (FAP) and subsequent peritoneal inclusion cysts. FAP is a rare, inherited condition connected with a defect in the APC gene. The main features are huge amounts of polyps, more than hundreds, growing in the colon and rectum since childhood. Polyps without treatment are correlated with nearly 100 percent risk of progression to colorectal cancer in the third or fourth decade of life. Surgical removal of the colon is the only effective treatment to prevent transformation into colorectal cancer. One of the methods is total proctocolectomy (TPC) with ileal pouch-anal anastomosis (IPAA) [7].

The patient was admitted to the Department of Obstetrics, Gynecology, and Oncology at the Medical University of Warsaw in March 2019 due to deterioration of her general condition, ascites, and lower abdominal pain. At 14, she underwent proctocolectomy with ileal pouch-anal anastomosis (IPAA) because of familial adenomatous polyposis. Nine years later she had enucleation of the right ovarian cyst with damage of the small intestine due to massive peritoneal adhesions. Then oral hormonal contraception was prescribed but the patient stopped it because of worse tolerance. In 2014 the patient suffered from the intensification of pain and after two attempts at surgical enucleation of the cyst, she underwent left adnexectomy. The adnexa were completely changed into a 3-locular cyst with a diameter of $20 \mathrm{~cm}$ (Fig. 1). In 2016 the patient complained of pain, and a multilocular cyst of $10 \mathrm{~cm}$ diameter with adhesions to the right adnexa and subacute intestinal obstruction was identified. Conservative treatment was conducted due to the lack of possibility of surgical treatment resulting in extensive adhesion at the small intestine loops with the parietal peritoneum. Six-fold drainage of recurrent peritoneal pseudocysts in the vicinity of the right ovary was done, obtaining 2-4.8 liters of fluid each time. Histological result revealed no cancer cells. In March 2019, the CT and MRI showed ascites and a 4-locular tumor, measuring $25 \times 17 \times 35 \mathrm{~cm}$, with a $2 \mathrm{~cm}$ thick capsule and multiple thin septations (Fig. 2). Because of the compression of the large tumor, bilateral 

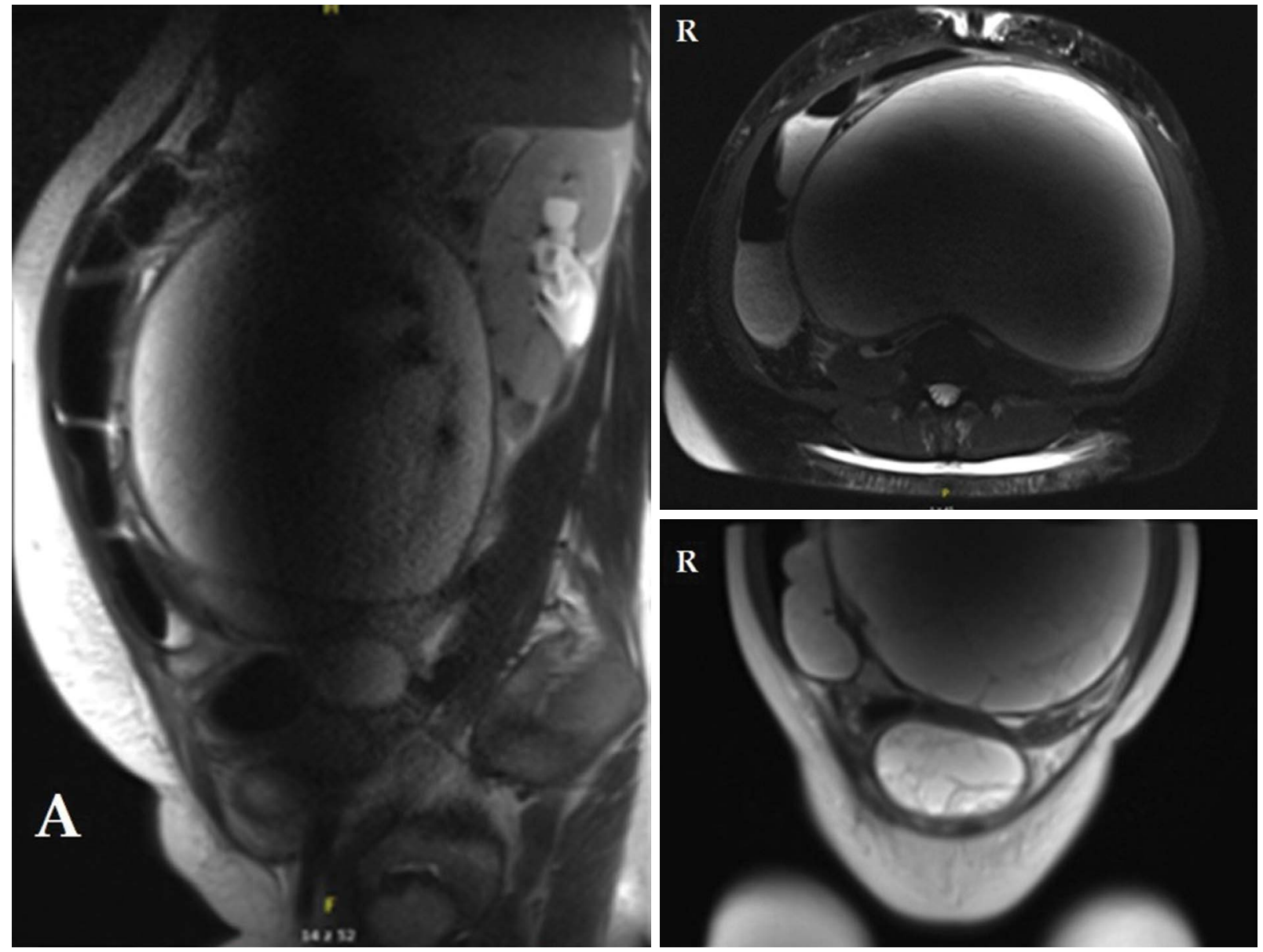

Figure 2. RMI scan 03.2019

hydronephrosis occurred. The abdominal cavity was punctured due to increased abdominal pain, obtaining four liters of bloody, cloudy fluid, without any tumor cells in the histopathology. Mini-laparotomy and 16-day suction drainage was used because of increasing ascites. The patient was discharged in good general condition without recurrence.

This case illustrates the potential recurrence of peritoneal inclusion cysts. Moreover, an ovarian mass and ascites in women of reproductive age are signs for a long list of differential diagnoses. Ascites may be the consequence of liver or renal failure, congestive heart failure, or some malignant diseases. Very often it is accompanied with endometriosis, ovarian hyperstimulation syndrome (OHSS), ectopic pregnancy, ruptured ovarian functional cyst, tuberculous peritonitis. Because of the documented correlation of ascites and benign ovarian fibroma, Meigs syndrome was described. However, all differential diagnoses should exclude ovarian cancer. In a large number of patients, careful history taking, and examination can provide important clues such as signs of chronic liver disease or history of operations in the case of PICs. Ultrasound, computed tomography scan, and magnetic resonance imaging can be helpful in confirming the diagnosis [8].
Even though a full spectrum of management options for patients with PICs remains controversial, both medical and surgical treatment methods are used, and there is no clear standard of treatment [3].

\section{Treatment of PICs}

Treatment of PICs depends individually on the patient's condition, symptoms, other diseases, and desire for procreation $[1,9]$.

PICs do not have malignant potential and spontaneously regress after menopause is observed [3]. The peak morbidity of PICs is in reproductive age. To alleviate intermittent pain, a combined hormonal oral contraceptive is chosen. It may decrease or stabilize the cyst volume and relieve symptoms by decreasing the production of cyst fluid [1, 3]. Another conservative method is image-guided aspiration, which allows fluid to be obtained for cytological examination and symptoms to be minimized because of minimal intervention and few complications. A combination of these two methods (aspiration and oral contraceptive) is often the most effective treatment [1] but due to the potential for recurrence of peritoneal inclusion cysts, most patients will have 
local reaccumulation of fluid. It is estimated that symptoms continue 3 to 28 weeks after the procedure [3]. That is why many patients choose surgical management. The operation can be concentrated on adhesiotomy or in a worse situation even bilateral adnexectomy and resection of pathological structures. The literature reports recurrence in up to 50\% of cases, but there is a lack of long-term follow-up data [2, 10]. Complete resection of all macroscopically visible cyst walls is definitive treatment. Indications for surgical excision are elevated CA-125, suspicion of malignancy in ultrasound view, recurrence of symptoms during conservative therapy [3] and decreasing level of quality of life.

Observation or hormonal therapy based on oral contraceptives can be a conservative method of treatment. On the other hand, surgical methods are sometimes the only way of treatment. Minimally invasive surgery is a better option due to fewer adhesions afterward than laparotomy, which can represent a protective factor in the future [10].

The benefits of minimally invasive surgery are the lower level of postoperative pain, a shorter time of recovery and better cosmetic effects. Disadvantages are a longer operating time and the technical difficulty of laparoscopic procedures caused by pelvic adhesion [10].

In contrast, the complication of PICs is infertility. Adhesions in the pelvis can lead to closure of the salpinx and difficulties in movements of a zygote. The size, location and formation processes of intraperitoneal adhesions are associated with changes in the pelvic anatomy and infertility, but there are cases of spontaneous conception occurring with a known peritoneal inclusion cyst [9]. Due to fact that the pregnancy is a hyperestrogenic state with rising human chorionic gonadotropin, the rapid increase and aggravation of symptoms can also be observed during pregnancy [3]. Mechanical compression also exacerbates symptoms. Cyst aspiration during the first trimester of pregnancy may prevent recurrence in advanced pregnancy and may be a valuable tool for managing peritoneal inclusion cysts during pregnancy [1].

Pregnancy seems to be a time in a woman's life of progesterone domination. PICs are rarely observed in this condition. However, every rapidly growing tumor during pregnancy should be diagnosed and treated. Fujimoto et al. in 2019 described a case of pelvic cysts during pregnancy, one of which was PICs [11].

Long-term use of combined hormonal oral contraceptives is undesirable in patients desiring fertility but discon- tinuing hormonal contraception may result in reaccumulation of peritoneal fluid and rapid growth of peritoneal inclusion cysts. Rapid growth and worsening of symptoms can also be observed during pregnancy.

\section{SUMMARY}

To conclude, peritoneal inclusion cysts should be considered in the differential diagnosis of abdominal pain multilocular cystic lesions or ascites in women after abdominal surgery. The final diagnosis is always based on the histological result, but the unknown etiology and pathogenesis of PICs do not allow a single best method of treatment to be established. This issue requires the continuation of multicenter, randomized clinical trials to find and standardize effective, personalized treatment for PICs.

\section{Conflict of interest}

The authors declare no conflict of interest.

\section{REFERENCES}

1. Jones SA, Salicco JM, Byers MS. Pelvic pain and history of previous pelvic surgery. Proc (Bayl Univ Med Cent). 2003; 16(1): 121-122, doi: 10.1080/08998280.2003.11927892, indexed in Pubmed: 16278726.

2. Singh A, Sehgal A, Mohan H. Multilocular peritoneal inclusion cyst mimicking an ovarian tumor: A case report. J Midlife Health. 2015; 6(1): 39-40, doi: 10.4103/0976-7800.153648, indexed in Pubmed: 25861208.

3. Vallerie A, Lerner J, Wright J, et al. Peritoneal Inclusion Cysts. Obstetrical \& Gynecological Survey. 2009; 64(5): 321-334, doi: 10.1097/ogx.0b013e31819f93d4.

4. Veldhuis WB, Akin O, Goldman D, et al. Peritoneal inclusion cysts: clinical characteristics and imaging features. Eur Radiol. 2013; 23(4): 1167-1174, doi: 10.1007/s00330-012-2695-8, indexed in Pubmed: 23263602.

5. Ho-Fung V, Jaimes $C E$, Pollock AN. Peritoneal inclusion cyst. Pediatr Emerg Care. 2011; 27(5):430-431, doi: 10.1097/PEC.0b013e3182184967, indexed in Pubmed: 21546811.

6. Sanges M, Pellegrini L, Imperatore $\mathrm{N}$, et al. Peritoneal inclusion cysts in Crohn's disease. Eur J Gastroenterol Hepatol. 2019; 31(8): 1070-1072, doi: 10.1097/MEG.0000000000001453, indexed in Pubmed: 31268900.

7. Galiatsatos P, Foulkes WD. Familial adenomatous polyposis. Am J Gastroenterol. 2006; 101(2): 385-398, doi: 10.1111/j.1572-0241.2006.0037 $5 . x$, indexed in Pubmed: 16454848.

8. Su MH, Cho SW, Kung YS, et al. Update on the differential diagnosis of gynecologic organ-related diseases in women presenting with ascites. Taiwan J Obstet Gynecol. 2019; 58(5): 587-591, doi: 10.1016/j. tjog.2019.07.002, indexed in Pubmed: 31542076.

9. Vallerie AM, Hsieh T, Baxi LV. Peritoneal inclusion cyst: effects on fertility and antepartum course. Obstet Gynecol. 2008; 112 (2 Pt 2): 498-500, doi: 10.1097/AOG.0b013e3181809e5c, indexed in Pubmed: 18669779 .

10. Lee SW, Lee SJ, Jang DG, et al. Comparison of laparoscopic and laparotomic surgery for the treatment of peritoneal inclusion cyst. Int J Med Sci. 2012; 9(1): 14-19, doi: 10.7150/ijms.9.14, indexed in Pubmed: 22211084.

11. Fujimoto $\mathrm{Y}$, Takahashi H, Horie K, et al. Rapid Growth of Pelvic Cyst during Pregnancy: A Case Report. Case Rep Obstet Gynecol. 2019; 2019: 3120921, doi: 10.1155/2019/3120921, indexed in Pubmed: 31214368. 